\title{
Una nota sobre los adjetivos de preferencia
}

\section{A note on preference adjectives}

\author{
ANTONIO FÁBREGAS \\ Universidad de Tromsø
}

Resumen. Los adjetivos de preferencia (favorito, preferido, predilecto) tienen propiedades gramaticales que los diferencian de otras clases de adjetivos, asimilables o no a los superlativos. Este trabajo propone un análisis de su comportamiento que se basa en la idea de que, frente a otros adjetivos, estos elementos encabezan estructuras léxicas de relación donde ellos son el núcleo de la estructura, no el sustantivo.

Palabras clave: adjetivos, posesivos, nombres de relación, superlativos, nuclearidad.

Abstract. Preference adjectives (favourite, preferred) have grammatical properties that contrast with those of other adjective classes, be they related to superlatives or not. This piece proposes an analysis of their behaviour based on the idea that, in contrast to other adjectives, these elements introduce relational structures where they, and not the related noun, are heads.

Keywords: adjectives, possessives, relational nouns, superlatives, headedness.

Data de recepción: 23-03-2020 - Data de aceptación: 27-05-2020. 


\section{EL PROBLEMA: EL COMPORTAMIENTO GRAMATICAL DE LOS ADJETIVOS DE PREFERENCIA}

Se ha observado recientemente en la bibliografía (Partee 2004, Boguslavsky 2009, Bosque 2019) que el adjetivo favorito tiene un comportamiento extraordinario entre otros modificadores adjetivales; dicho comportamiento, como veremos, se extiende a otros adjetivos semánticamente próximos a él (1).

\section{(1) favorito, preferido, predilecto}

Son varias las propiedades especiales observadas para estos adjetivos, que de ahora en adelante llamaremos 'adjetivos de preferencia'. Los tres adjetivos, para empezar, introducen un posesivo (2a) que se interpreta semánticamente como la entidad necesariamente animada para la que lo denotado por el sustantivo es preferido. Que la relación semántica del posesivo es establecida con el adjetivo y no con el sustantivo se puede comprobar en el hecho de que (2a) no entraña (2b) (Bogulavsky 2009, Bosque 2019: 18).

(2) a. mi película \{favorita / preferida / predilecta\}

b. $\nRightarrow$ mi película

En efecto, podemos hablar de nuestra película favorita sin que esa película sea nuestra en ninguna de las interpretaciones semánticas que establece el posesivo con el sustantivo (Rigau \& Picallo 1999). Con otros adjetivos fuera de estas clases, el entrañamiento se produce (3) sencillamente porque el posesivo establece una relación semántica con el sustantivo, no con el adjetivo.

(3) a. mi película \{galardonada / exitosa / fracasada

b. $\Rightarrow$ mi película

El posesivo en (2a), de hecho, se interpreta como un experimentante psicológico, la entidad que evalúa la entidad como preferible, por lo que siempre tiene la misma relación semántica con el adjetivo.

En segundo lugar, como observa Partee (2004), los adjetivos de preferencia convierten al sustantivo al que modifican en uno que expresa una relación, independientemente de que antes lo fuera o no. Específicamente, aunque el sustantivo solo no se asocie semánticamente a un participante, cuando se agrega el adjetivo de preferencia es necesario que se interprete que hay un experimentante que evalúa un conjunto de 
entidades de la que se extrae aquella que considera predilecta. Concentrémonos en la interpretación de (4).

(4) mi libro favorito

Una interpretación semántica completa de (4) implica dos propiedades que el sustantivo libro por sí solo no implica. En primer lugar, se debe interpretar que existe un participante que es quien somete las entidades descritas a evaluación. En segundo lugar, necesariamente, el referente del sustantivo debe extraerse de un conjunto mayor de entidades definidas, relevante dentro del discurso, del cual se toma una sola que se identifica como la que prefiere el hablante. De hecho, si el sustantivo designa una entidad única por cuestiones conceptuales, agregar estos adjetivos tiene un efecto marcado semánticamente, como sucede en (5).

\section{(5) \#mi sol favorito}

Necesariamente, el hablante tiene que estar pensando en una situación donde existe un grupo de soles entre los cuales se resalta uno como aquel por el que muestra preferencias. Podría pensarse que este papel se sigue necesariamente de la posición pospuesta del adjetivo, pero nótese que no cabe decir que un sustantivo que designa a una entidad única rechaza los adjetivos pospuestos (este sol embriagador, el sol luminoso...). Con este adjetivo es imprescindible que el participante al que corresponde el posesivo evalúe dentro de un conjunto de entidades de la misma clase. El carácter relacional del sintagma nominal viene de que el adjetivo introduce un participante que actúa como el evaluador de la preferencia, pero esa preferencia necesariamente ha de manifestarse sobre un conjunto definido de entidades, de la que se extrae una.

Estos adjetivos no están rechazados por todos los sustantivos que, en principio, designan entidades únicas. Con sustantivos que expresan papeles sociales, es posible interpretar distintas entidades que han desempeñado dicho papel a lo largo del tiempo (mi Papa favorito, mi marido favorito), pero la presencia de un adjetivo de preferencia fuerza siempre la presuposición de que la entidad denotada por el sustantivo que se destaca es solo una dentro de un grupo posible.

Como ya observa Bosque (2019: 19), tras esta interpretación en que se extrae un referente dentro de un grupo presupuesto se encuentra que, al menos en su significado, los adjetivos de preferencia se asimilan a los superlativos (el chico más alto de la clase) al menos en el sentido de que presuponen la comparación de una entidad con otras de su mismo grupo para seleccionar aquella que tiene un grado más alto de 
cierta propiedad. Estos adjetivos, pues, sirven para designar al miembro del grupo hacia el que una entidad dirige sus preferencias por encima de los otros miembros. De hecho, los adjetivos de preferencia permiten la introducción sintáctica de sintagmas preposicionales que denotan el grupo general del que se extrae la entidad favorita.

(6) a. mi asignatura \{favorita / predilecta / preferida\} entre todas las asignaturas b. mi día \{favorito / predilecto / preferido\} de todos los días

Bosque (2019: 20) observa, finalmente, que estos adjetivos son los únicos que permiten la combinación directa con el posesivo, sin un sustantivo explícito. Aunque Bosque hace esta observación para favorito (7a), esta misma combinación se documenta para los otros miembros de la clase, y es imposible en el resto de adjetivos (8).

(7) a. mi favorito

b. Este diseño es mi preferido. [Pinterest]

c. Este es mi hijo, el amado, mi predilecto [Mateo 3: 16]
[M

(8) *mi $\{$ gris / bueno / elegante / notorio / biológico $\}$

Este comportamiento gramatical especial sugiere que esta clase de adjetivos ha de recibir un análisis diferente a los modificadores adjetivales normales. En la siguiente sección presentaremos el análisis sintáctico de Bosque (2019), y explicaremos qué desventajas tiene, desde nuestro punto de vista. La alternativa al análisis de Bosque se presenta en $\S 3$.

\section{ANÁLISIS PREVIOS}

No son muchos los análisis que se han propuesto. Partee (2004) proporciona un análisis semántico que se concentra en dar cuenta del hecho de que los adjetivos de preferencia fuerzan una lectura de relación del sustantivo al que modifican — por lo que propone que estos modificadores son operadores que convierten el sustantivo en uno de relación, de forma semejante a como lo hacen los superlativos-. El único análisis sintáctico explícito que se ha propuesto se debe a Bosque (2019: 20-21). El análisis de Bosque se apuntala sobre tres ideas: en primer lugar, el posesivo está introducido por el adjetivo, como un complemento preposicional (9a). Como es fácil de entender, si el adjetivo es, como se asume generalmente, un modificador no obligatorio del núcleo nominal, el posesivo que introduce no debería nunca pasar a ser un determinante de todo el sintagma nominal. Por este motivo, la segunda idea que 
forma parte del análisis de Bosque es que se produce un reanálisis por el que el adjetivo de preferencia y el sustantivo al que modifican pasan a ser un núcleo complejo (9b). Una vez reanalizado como parte del núcleo, el complemento del adjetivo pasa a ser un complemento del núcleo, y por lo tanto ya puede funcionar como el determinante de toda la estructura $(9 \mathrm{c})$.
(9) a. [color] [favorito de María]
b. [color favorito] [de María]
c. [su] [color favorito]

La tercera idea que forma parte de esta propuesta es que este reanálisis se produce en el caso de estos adjetivos, y no de otros, por la relación semántica que los adjetivos de preferencia tienen con los superlativos (10). La idea es que los superlativos también introducen un complemento (10a) y se reanalizan con el verbo (10b) formando un núcleo complejo.

(10) a. [era] [el mejor de ellos]

b. [era el mejor] [de ellos]

Pese a que el análisis de Bosque tiene la ventaja de proponer una estructura sintáctica que explique por qué el adjetivo de preferencia no funciona como un modificador normal, y por lo tanto está posiblemente bien encaminado, creemos que se enfrenta a algunos problemas que lo ponen en duda. Aunque, como suele suceder, los argumentos empíricos tienen más fuerza que los teóricos, podemos anotar al margen que el reanálisis tiene un estatuto teórico dudoso en las teorías actuales. Entendido en sus términos clásicos, el reanálisis sería una operación semántica que de alguna manera afecta al estatuto sintáctico de los elementos, algo que presupone un sistema en que la interpretación semántica no es un resultado inmediato de la estructura sintáctica, sino que la condiciona. Si el reanálisis se reformula en términos sintácticos, como un movimiento sin reflejo en la forma fonológica, el problema es que dicho movimiento no implica cambios en la estructura linear de los constituyentes, y en caso de ser movimiento de núcleos, no estaría impedido por proyecciones funcionales, cosa que suele ser el caso en otros ejemplos clásicos de esta operación.

Pero centrémonos sobre todo en los argumentos empíricos y sus predicciones. La propuesta de que el adjetivo y el sustantivo en (9b) forman un núcleo complejo implica que el posesivo pasa a ser un complemento de los dos. Sin embargo, la presencia de ese complemento del adjetivo no impide que el sustantivo introduzca su 
propio complemento (11a), que puede convertirse en posesivo sin problemas (11b), conviviendo incluso con la posesivización del complemento del adjetivo para resultar en una estructura nominal con dos posesivos (11c), algo que es generalmente imposible en otros contextos $(10 \mathrm{~d})$.

(11) a. el poema de Lorca favorito de Luisa

b. su poema de Lorca favorito

c. su poema favorito \{suyo / mío / tuyo / nuestro / vuestro \}

d. *su poema mío

Esto sugiere que el adjetivo y el sustantivo siguen manteniendo independencia estructural suficiente para introducir cada uno un posesivo diferente; no está claro cómo se compadece esto con la idea de que el reanálisis convierte el complemento del adjetivo en complemento del sustantivo. Asimismo, no está claro en qué sentido el adjetivo de preferencia y el sustantivo se han reanalizado dentro del mismo núcleo: si formar un núcleo complejo ha de tomarse literalmente como una reorganización sintáctica, esperamos que adjetivo y sustantivo deban ser adyacentes uno a otro. Sin embargo, es posible interponer entre ambos otros constituyentes sintácticos.

(12) a. mi abrigo [rojo y elegante] favorito

b. mi reloj [de pulsera] favorito

c. mi viaje [al extranjero] favorito

Si el reanálisis se hubiera producido en un sentido sintáctico (Baker 1988, 1996), esperaríamos que fueran obligatorios abrigo favorito rojo y elegante, reloj favorito de pulsera y viaje favorito al extranjero; de hecho, estas secuencias son más marcadas que las de (12). Estos datos sugieren en primer lugar que los modificadores nominales restrictivos, preposicionales o no, deben combinarse con el núcleo nominal de forma más directa que los adjetivos de preferencia, y además hacen dudar más aún de la idea de que los adjetivos de preferencia sean asimilables a los adjetivos relacionales: los adjetivos relacionales son más internos al sustantivo que los calificativos y los sintagmas preposicionales.

La idea de que los adjetivos de preferencia deben asociarse con los superlativos tampoco está exenta de problemas. Una propiedad de los adjetivos superlativos es que, frente a los comparativos (Sáez del Álamo 1993), pueden aparecer en posición prenominal. Esto se aplica tanto a los superlativos gramaticales (13c) como a los adjetivos que, sin serlo gramaticalmente, se pueden asimilar a ellos, como último y primero (Eguren y Fábregas 2006). 
(13) a. la última novela de Marías

b. la primera novia de Juan

c. la más importante pregunta de Pedro

En contraste, ninguno de los adjetivos de preferencia permite de forma natural la posición prenominal cuando introducen un genitivo, como se ve en (14). De hecho, la posición fija de estos adjetivos es otra propiedad que debe explicarse en el análisis, algo que una propuesta de reanálisis no hace de forma suficiente.

(14) *mi \{favorita / preferida / predilecta $\}$ novela

Tampoco se pueden asimilar los adjetivos de preferencia a los superlativos con respecto a la interacción con la definitud. Los superlativos (15) no admiten los sintagmas nominales indefinidos. En el caso de los adjetivos asimilables a ellos, como primero y último, al menos en posición posnominal son imposibles los indefinidos - mientras que en posición prenominal pueden llegar a admitirlos, como en un último deseo o un primer borrador-

(15) a. *una novela última

b. *una novela primera

c. *un chico más alto de la clase

Los adjetivos de preferencia, en posición posnominal, no tienen problema alguno en combinarse con sintagmas nominales indefinidos, como muestra (16), con datos tomados del Corpus del Español de Mark Davies.

(16) a. El juego fue, pues, para Quiroga, una diversión favorita y un sistema de expoliación [Facundo, Domingo Faustino Sarmiento]

b. debido al encanto natural que lo rodea se ha convertido en un centro favorito de clase alta [Herald, 8 de noviembre 1998]

c. es previsible que el país se mantenga como un destino favorito de los capitales foráneos [Yucatán, 28 de junio de 1997]

d. Siempre habrá una canción favorita que logrará mucho en la reacción del bebé [mibebitoyyo [blog], Bolivia]

Si bien no despliegan el comportamiento gramatical de los superlativos, los adjetivos de preferencia sí tienen propiedades especiales con respecto a la gradación. Específicamente, cuando introducen un posesivo, no admiten la combinación con gradadores comparativos (17a), de grado positivo (17b) o superlativos (17c). 
(17) a. *mi novela más favorita

b. *mi novela muy favorita

c. *mi novela favoritísima

De hecho, si combinamos la imposibilidad de graduar estos adjetivos con el hecho de que su posición gramatical es obligatoriamente posnominal, más que asimilarse a los superlativos parecería que estos adjetivos se asimilan a los adjetivos relacionales (Bosque 1993). La diferencia con los adjetivos relacionales, sin embargo, es que los adjetivos de preferencia son externos a los calificativos. En (18) vemos que un adjetivo relacional debe ser más interno al sustantivo que un adjetivo calificativo, mientras que en (19) vemos que los adjetivos de preferencia han de ser más externos que los adjetivos calificativos, y de hecho prefieren ir tras los otros modificadores. Recuérdense en este sentido los ejemplos de (12), donde era posible introducir sintagmas preposicionales entre el sustantivo y el adjetivo de preferencia.

(18) a. su prima francesa simpática

b. *su prima simpática francesa

(19) a. ??su prima favorita simpática

b. su prima simpática favorita

No es claro, tampoco, cómo un análisis en términos de reanálisis explicaría las similitudes con los adjetivos relacionales y la posición especial que tienen los adjetivos de preferencia dentro del sintagma nominal. Pasemos, pues, a nuestra propuesta acerca de su análisis.

\section{UN ANÁLISIS ALTERNATIVO}

Antes de presentar el análisis, volvamos un segundo atrás para centrarnos en la dificultad fundamental que producen estos adjetivos de preferencia. Los adjetivos de preferencia introducen un complemento preposicional, correspondiente a la persona que evalúa el sustantivo, y ese complemento del adjetivo puede convertirse en un posesivo de todo el sintagma nominal. Si suponemos una estructura en que el adjetivo es un modificador adjunto o, como se representa en (20), un especificador de una proyección funcional (Cinque 2010), sería imposible extraer el complemento del SA, por estar dentro de un dominio sintácticamente cerrado (al menos, desde Ross 1967). 
(20)

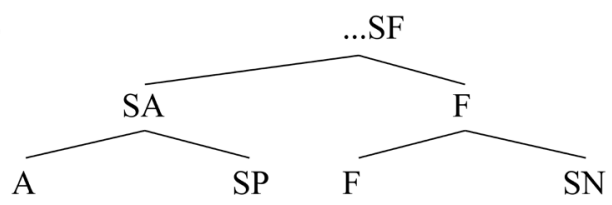

favorita de Juan princesa

La propuesta de reanálisis de Bosque (2019) en el fondo trata solo de obtener una configuración en la que el adjetivo está en posición de núcleo y por lo tanto el complemento no queda encerrado en un dominio del que es imposible extraerlo. Ya hemos visto, sin embargo, que esto tiene problemas empíricos y no proporciona explicaciones sobre, entre otras cosas, la posición posnominal obligatoria del adjetivo de preferencia o la imposibilidad de graduarlo.

La estructura de (21) presenta nuestra propia propuesta.

(21)

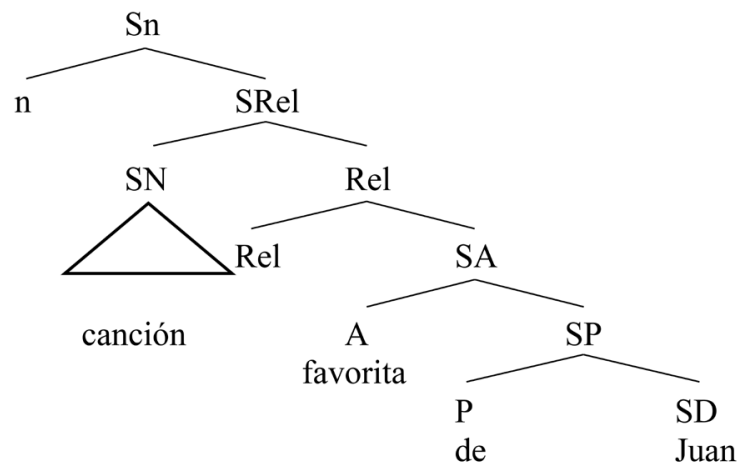

La base de nuestra propuesta es que un adjetivo de preferencia introduce una proyección relacional (SRel). Esta proyección relacional tiene una denotación que corresponde a la función semántica subespecificada $\mathrm{R}$, que establece una relación de cualquier tipo entre el complemento y el especificador, dejando que el contenido semántico conceptual de los dos constituyentes determine el contenido de dicha relación. El núcleo Rel toma la estructura del adjetivo (SA) como complemento, y sitúa en el especificador el sustantivo léxico (SN). El SA introduce el complemento preposicional, que puede ser extraído como posesivo porque está en posición de complemento dentro de la estructura. De esta manera, el participante que evalúa un grupo de entidades es parte del primer miembro de la relación (dentro de SA) y el conjunto de entidades del que se extrae el elemento favorito es el segundo miembro, como el SN que aparece en la posición de especificador del SRel. Dado que el adjetivo 
de preferencia tiene valor modal e introduce un participante que actúa como evaluador, este complemento determina que la función $\mathrm{R}$ sea una de preferencia dentro de un conjunto de unidades.

Nuestro análisis tiene dos ingredientes fundamentales: la propuesta de que a la estructura subyace un núcleo relacional y la propuesta de que en ella el sustantivo está dividido y no es el núcleo de la estructura. Examinemos por turnos tanto las bases teóricas como las consecuencias de cada uno de estos dos aspectos.

Partee (2004) nota que la presencia de un adjetivo de preferencia automáticamente fuerza una lectura de relación del sustantivo; esto es lo que explica que el SN esté introducido como el segundo miembro de SRel. Tomamos de Mateu (2002) — cf. también Hale \& Keyser (2002) — la idea de que un núcleo relacional es un elemento subespecificado categorialmente cuyo papel es el de definir una relación sin valor semántico rígido entre dos entidades. En este caso, Rel es el responsable de que se fuerce una lectura en la que el sustantivo designa un individuo que, de entre una clase de objetos, es destacado como el favorito. Es necesario, pues, que el sustantivo designe una clase de entidades en este nivel, de manera que esa clase pueda ser evaluada relacionalmente en virtud de la preferencia que tenga una entidad animada, el complemento preposicional de SA.

Por este motivo, es crucial que el sustantivo que se relaciona dentro de la estructura introducida por el adjetivo de preferencia exprese una clase de entidades (Carlson 1977, Zamparelli 2000, Baker 2002, entre otros), no un individuo específico. Por ese motivo, seguimos a Baker (2002), entre otros, en la propuesta de que un sustantivo se divide al menos en dos capas: una parte léxica, predicacional, en la que designa el conjunto de propiedades que definen a la clase general de entidades que representa (SN), y una capa funcional (Sn) en la que se agrega un índice de identidad a las propiedades definidas en SN como un paso previo a definir un individuo que represente a la clase general de entidades. El fragmento de estructura relevante para esta parte del análisis está destacado en (22).

(22) $\left[_{\mathrm{Sn}} \quad \mathrm{n}\left[\left[_{\text {SRel }}[\mathrm{SN}] \quad\right.\right.\right.$ Rel $\left.\left.[\mathrm{SA}]\right]\right]$

La construcción relacional contiene solo la parte predicacional, y es seleccionada por una proyección nominal funcional, Sn. Composicionalmente, esto implica asignar el índice de identidad ( $\mathrm{Sn}$ ) a la entidad que, dentro de la clase de entidades que designa $\mathrm{SN}$, es la que se define como favorita (para alguien). De esta manera, explicamos la semántica relacional que los adjetivos de preferencia imponen a todo sustantivo. 
No solamente explicamos esto. En esta estructura, explicamos la posición rígida del adjetivo de preferencia. Recordemos que ese adjetivo debe estar en una posición postnominal y que sigue a otros modificadores del sustantivo. Esto es justo lo que esperamos de la estructura: al ser el complemento de un SRel que toma al SN como especificador, debe seguirlo necesariamente en virtud del Axioma de Correspondencia Lineal (Kayne 1994), porque SN y Sn lo mandan-c. Además, ya que SN, con toda su parte predicacional, está introducido en el especificador, esperamos que cualquier otro modificador o complemento de SN preceda al adjetivo, como vimos en (12). El diagrama de (23) muestra esto.

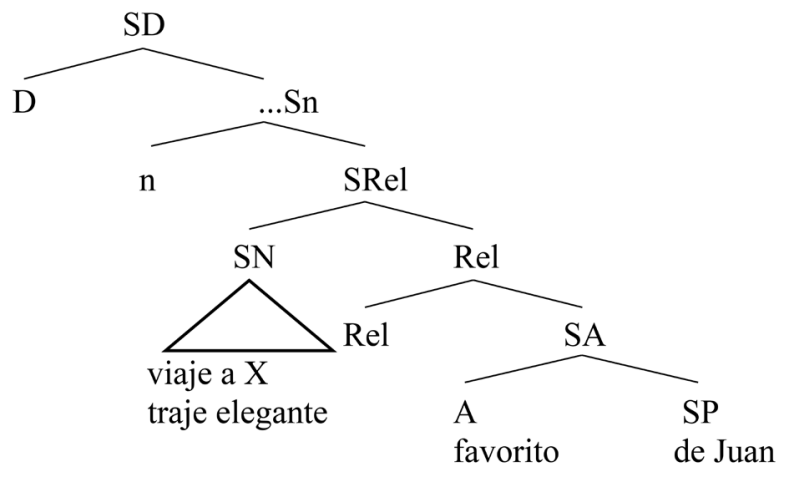

La imposibilidad de combinar los adjetivos de preferencia con el grado, proponemos, también se sigue de aquí: frente a otros modificadores — pero tal vez igual que los adjetivos relacionales (cf. Bosque 1993, Fábregas 2007) — los adjetivos de preferencia introducen Rel encima de su capa léxica en lugar de los núcleos cuantificacionales o referenciales que corresponden al grado en el adjetivo (Corver 1997).

Obsérvese que en nuestro análisis es el SN el constituyente que se encuentra en una posición de especificador. Predecimos, pues, que son los complementos del sustantivo los que no podrán ser extraídos precisamente porque se encuentran atrapados dentro del dominio sintáctico cerrado que define el especificador. Creemos que esta predicción se cumple. Véase el contraste de (24) frente a (25).

(24) su reforma

a. su reforma educativa $(\mathrm{su}=$ agente $)$

b. su reforma por parte de $\mathrm{X}(\mathrm{su}=$ paciente $)$ 
(25) su reforma favorita mía

a. la reforma de las que ha hecho él que yo prefiero

b. *la reforma que le ha afectado a él que yo prefiero

El posesivo en (24) admite dos lecturas (cf. Escandell 1995): puede corresponder al agente de la reforma (24a) o al paciente, la entidad reformada (24b). Sin embargo, cuando se combina con un adjetivo de preferencia (25), la única lectura posible que se admite es aquella en que el posesivo es la entidad que desarrolla la reforma, no el paciente (25a vs. 25b) —nótese que eliminamos la lectura del posesivo como la entidad que valora al añadir el segundo posesivo dependiente de favorito-. ¿Qué explica este contraste? Siguiendo a Scholten (2018), solo los argumentos internos de un sustantivo - derivado o no- se introducen en el interior del SN (26). Los argumentos externos, correspondientes al agente o al posesor estándar, se introducen en Sn o tal vez en una proyección funcional aún más alta, correspondiente al número (Ritter 1991) o ciertos determinantes (Abney 1987).

(26)

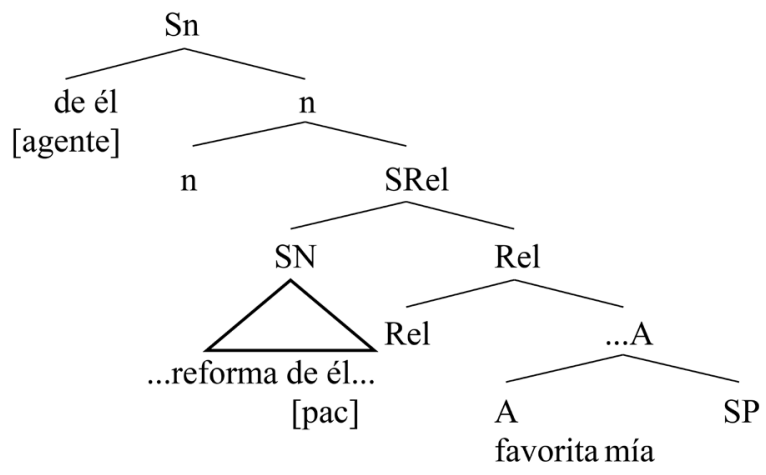

De esta manera, no es posible posesivizar un complemento paciente porque este se encuentra en SN, que en la estructura impuesta por el adjetivo de preferencia aparece en una posición de especificador; los agentes sí pueden posesivizarse porque están introducidos por núcleos que proyectan en la estructura.

Nuestra estructura, por ello, permite explicar sin problemas la presencia de dos posesivos. El posesivo prenominal en (27) procede de Sn u otra proyección funcional de N, mientras que el segundo posesivo es el introducido por SA desde la posición de núcleo. Seguimos a Brucart (1994) en la propuesta de que el posesivo prenominal está en posición de especificador de $\mathrm{SD}$, mientras que el posnominal funciona como un adjetivo que aparece en posición baja. 
(27)

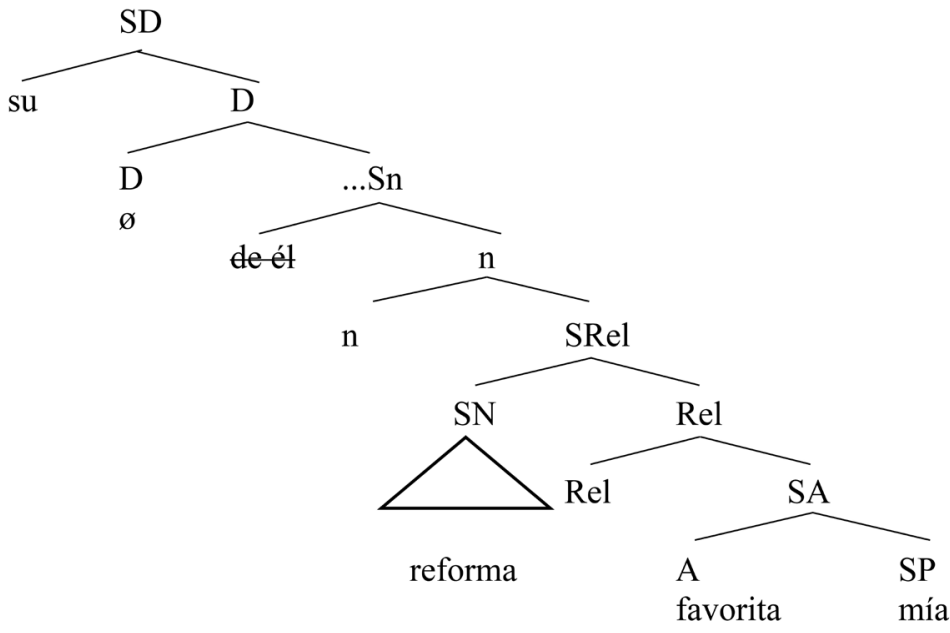

Finalmente, la estructura también permite explicar por qué son solo los adjetivos de preferencia los que permiten la combinación directa con el posesivo prenominal (cf. 7). Comparemos en este sentido la estructura de (27), resumida como (28a), con la estructura en que el adjetivo es un modificador adjunto o especificador del SN (28b).
a. $\left[_{\mathrm{SD}}[\mathrm{su}] \mathrm{D}\left[\mathrm{Sn}_{\mathrm{Sn}}\right.\right.$
n $\quad$ SRel $_{\text {sRel }}$
[SN]
Rel
[SA]]]]
b. $\left[_{\mathrm{SD}} \quad[\mathrm{su}] \mathrm{D}\left[\left[_{\mathrm{Sn}}\right.\right.\right.$
n $\quad\left[_{\mathrm{SF}}\right.$
[SA] F
[SN]]]]

Como se observa en (28a), el adjetivo está en la espina dorsal de la cláusula, como un núcleo que proyecta la etiqueta, mientras que en (28b) es un especificador en una estructura donde SN es el núcleo. Cuando SN se elide de la estructura (29), en (29a) el material que permanece está en la espina dorsal de la cláusula, mientras que en (29b) es un adjunto o especificador.
(29)
$\begin{array}{lll}\text { a. }\left[_{\mathrm{SD}}\right. & {[\mathrm{su}] \mathrm{D}} & {[\mathrm{Sn}} \\ \text { b. } *[\mathrm{su}] \mathrm{D} & {[\mathrm{S}}\end{array}$
n
$\Gamma_{\text {SRel }}$
[SN]
Rel
[SA]]]]

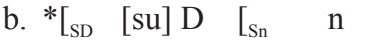
$[\mathrm{SF}$
[SA] F
[SN]]]

Dicho de otra manera: con los adjetivos de preferencia, elidir el SN es elidir un elemento no nuclear, como si se elidiera el adjetivo en (28b). Si el posesivo prenominal necesita que se mantenga el material nuclear dentro del SD para poder aparecer, el contraste de (7) frente a (8) queda explicado de forma directa: el adjetivo es el núcleo si hablamos de un adjetivo de preferencia. 


\section{CONCLUSIONES}

Este artículo ha propuesto que los adjetivos de preferencia tienen propiedades especiales porque introducen estructuras relacionales de las cuales son complementos, convirtiendo así al SN en un especificador dentro de la estructura que está por debajo de las proyecciones funcionales del sustantivo. De esta manera, la única relación que tienen estos adjetivos con los superlativos es semántica: extraer de un conjunto de entidades (denotado por SN) un individuo (asignado por Sn) que para alguien es el mejor dentro del conjunto.

Esta configuración explica, entre otras cosas, la combinación con el posesivo y sin sustantivo, las restricciones interpretativas de los posesivos introducidos por el sustantivo, la posición fija de estos adjetivos y su incapacidad para ser modificados en grado.

Resulta tentador extender esta estructura, $\mathrm{u}$ otras semejantes, a los adjetivos que introducen complementos indirectos que pueden convertirse en pronombres del verbo, como en (30).

(30) a. ser fiel a alguien

b. serle fiel

No obstante, estas estructuras adjetivales no tienen las mismas propiedades - por ejemplo, no introducen posesivos por sí mismos, ya que su amigo fiel implica su amigo - , por lo que deberán ser estudiadas en otros trabajos. Esperamos, sin embargo, haber proporcionado al menos una explicación clara de las propiedades de los adjetivos de preferencia.

\section{BIBLIOGRAFÍA}

ABNey, S. (1987): The English noun phrase in its sentential aspect. Tesis doctoral, MIT. BAKER, M. C. (2002): Lexical categories: nouns, verbs and adjectives. Cambridge: Cambridge University Press. https://doi.org/10.1017/CBO9780511615047

BogusLavsky, I. (2009). "Enlarging the diversity of valency instantiation patterns and its implications", en P. Bosch et alii (eds.): Logic, language and computation. Berlin: Springer, pp. 206-220. https://doi.org/10.1007/978-3-642-00665-4_17

Bosque, I. (1993): "Sobre las diferencias entre los adjetivos relacionales y los calificativos", Revista Argentina de Lingüistica 9, pp. 9-48. 
Bosque, I. (2019): "Sobre la naturaleza composicional de las unidades gramaticales", en D. García Padrón et alii (eds.): Clases y categorías en la semántica del español y sus interfaces. Berlin: De Gruyter, pp. 11-26. https://doi. org/10.1515/9783110637700-002

BRUCART, J. M. (1994): "Incompatibilidad entre posesivos y relativas especificativas", en V. Demonte (ed.): Gramática del español. México: Colegio de México, pp. 11-26.

CARLson, G. C. (1977): Reference to kinds in English. Tesis doctoral, University of Massachusetts, Amherst.

CinQue, G. (2010): The syntax of adjectives. Cambridge (Mass.): MIT Press. https:// doi.org/10.7551/mitpress/9780262014168.001.0001

Corver, N. (1997): “Much-support as last resort”, Linguistic Inquiry 28, pp. 119-164.

Eguren, L. y A. FÁBregas (2006): “Cuestiones de orden: semántica y sintaxis de los adjetivos ordinales", Estudios de Lingüística de la Universidad de Alicante 19, pp. 85-105.

EsCANDELl, M. V. (1995): Los complementos del nombre. Madrid: Arco / Libros.

FÁBREGAS, A. (2007): "The internal syntactic structure of relational adjectives", Probus 19, pp. 1-36. https://doi.org/10.1515/PROBUS.2007.001

Hale, K. y Keyser, S. J. (2002): Prolegomena to a theory of argument structure. Cambridge (Mass.): MIT Press. https://doi.org/10.7551/mitpress/5634.001.0001

KAYNE, R. S. (1994): The antisymmetry of syntax. Cambridge (Mass.): MIT Press.

MATEU, J. (2002): Argument structure: relational construal at the syntax-semantics interface. Tesis doctoral, Universitat Autònoma de Barcelona.

Partee, B. H. (2004): Compositionality in formal semantics. Oxford: Blackwell. https://doi.org/10.1002/9780470751305

Rigau, G. \& Picallo, C. (1999): "El posesivo y las relaciones posesivas", en I. Bosque y V. Demonte (dirs.): Gramática descriptiva de la lengua española. Madrid: Espasa-Calpe, pp. 973-1023.

Ritter, E. (1991): "Two Functional Categories in Noun Phrases: Evidence from Modern Hebrew", en S. Rothstein (ed.): Syntax and Semantics 25: Perspectives on Phrase Structure. New York: Academic Press, pp. 37-62. https://doi. org/10.1163/9789004373198_004

Ross, J. (1967): Constraints on variables in syntax. Tesis doctoral, MIT.

Scholten, J. (2018): The ins and outs of external possession. Utrecht: LOT.

ZAMPARELLI, R. (2000): Layers in the determiner phrase. Nueva York: Garland. 